\title{
Vascular Ehlers-Danlos syndrome in 2 Polish patients: identification of 2 novel COL3A1 gene mutations
}

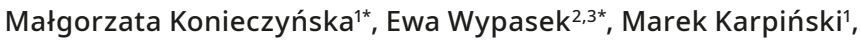 \\ Monika Komar ${ }^{1,4}$, Sofie Symoens ${ }^{5}$, Paul J. Coucke ${ }^{5}$, Anetta Undas ${ }^{6,7}$ \\ 1 Department of Diagnostics, John Paul II Hospital, Kraków, Poland \\ 2 Laboratory of Molecular Biology, John Paul II Hospital, Kraków, Poland \\ 3 Faculty of Medicine and Health Sciences, Andrzej Frycz Modrzewski Krakow University, Kraków, Poland \\ 4 Department of Cardiac and Vascular Diseases, Institute of Cardiology, Jagiellonian University Medical College, Kraków, Poland \\ 5 Center for Medical Genetics Ghent, Ghent University Hospital, Department of Biomolecular Medicine, Ghent, Belgium \\ 6 Department of Cardiac Surgery, Anesthesiology and Experimental Cardiology, Institute of Cardiology, Jagiellonian University Medical College, Kraków, Poland \\ 7 Krakow Center for Medical Research and Technology, John Paul II Hospital, Kraków, Poland
}

Correspondence to: Ewa Wypasek, PhD, Laboratory of Molecular Biology, John Paul II Hospital, ul. Prądnicka 80, 31-202 Kraków, Poland, phone: +48126143004 , email: ewa.wypasek@wp.pl Received: August 23, 2019. Revision accepted: September 29, 2019 Published online: October 1, 2019. Kardiol Pol. 2019;

77 (11): 1070-1073 doi:10.33963/KP.15005 Copyright by the Author(s), 2019

*MK and EW contributed equally to this work.
Introduction The Ehlers-Danlos syndrome (EDS) is a genetically and clinically heterogeneous disorder involving joint hypermobility, skin hyperextensibility, and tissue fragility. ${ }^{1}$ According to the 2017 international classification, ${ }^{2}$ EDS is divided into 13 types. Vascular EDS (vEDS) is the most severe form inherited in an autosomal dominant pattern and characterized by an increased risk of sudden death due to the rupture of large arteries, gastrointestinal perforation, or uterine rupture during pregnancy (for criteria, see Supplementary material, Table S1). Additional symptoms defining this syndrome are thin translucent skin with prominent subcutaneous vessels, easy bruising, small joint hypermobility, early-onset varicose veins, and tendon or muscle rupture. ${ }^{1}$ The minimal criteria suggestive of vEDS (ie, family history of the disorder, arterial rupture or dissection in individuals younger than 40 years of age, unexplained sigmoid colon rupture, or spontaneous pneumothorax in the presence of other features consistent with vEDS) should all lead to diagnostic workup to verify if the clinical diagnosis could be confirmed by genetic testing. Molecular testing for vEDS should also be considered in the presence of a combination of the other "minor" clinical features listed in Supplementary material, Table $S 1 .^{2}$

Vascular EDS is usually caused by mutations in the collagen type III alpha I chain (COL3A1) gene, mostly heterozygous missense mutations, typically glycine substitutions in the Gly-X-Y repeat, or splice-site variants affecting the triple helical sequence leading to altered type III collagen synthesis and assembly. ${ }^{2,3}$ As a consequence of a molecular defect in the COL3A1 gene, resistance to mechanical stress of the arteries, bowel, and uterus is decreased. ${ }^{4}$ Women with vEDS have an increased risk of obstetric complications including uterine rupture and dissection of major arteries and veins. ${ }^{5}$

To our knowledge, we report the first 2 Polish patients with vEDS in whom new causal mutations have been detected.

Case 1 A 33-year-old woman (height, $158 \mathrm{~cm}$; weight, $48 \mathrm{~kg}$ ) was referred to John Paul II Hospital, Kraków, Poland, for genetic workup. At the age of 2, she underwent Achilles tenotomy due to clubfoot, and at the age of 29, she underwent stent implantation for the spontaneous right common iliac artery aneurysm and dissection. At the age of 32, she experienced spontaneous sigmoid colon perforation and underwent colostomy with partial colectomy. She was pregnant twice and gave birth by uneventful vaginal delivery. Currently, computed tomography angiography showed that the ascending and descending aortic segments were not dilated; the maximum diameter of the abdominal aorta at the level of the bifurcation was $20 \mathrm{~mm}$, along with dilation 

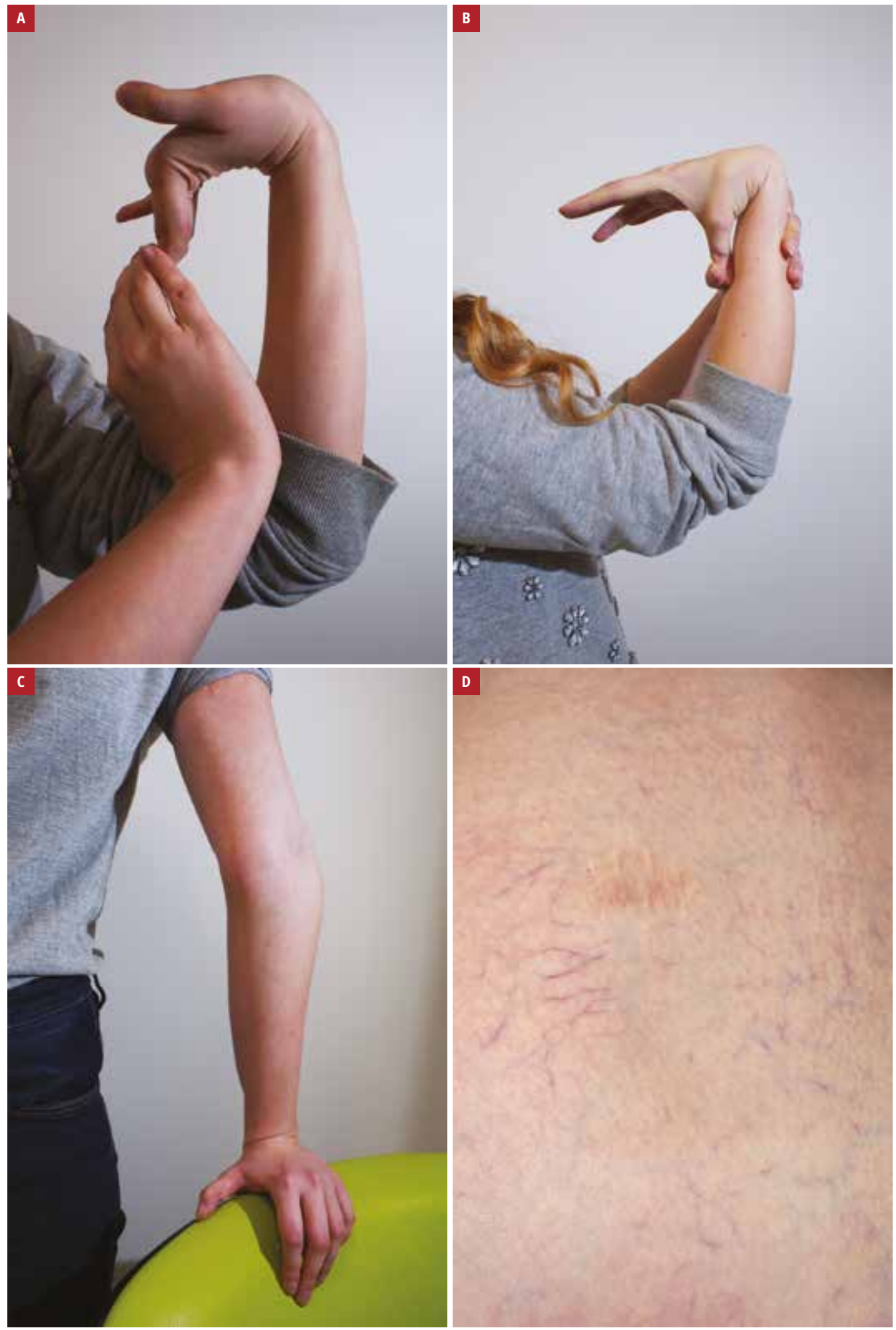

FIGURE 1 Case 2. Joint hypermobility in the fingers (A) and thumb (B), elbow bending (C), as well as translucent skin with visible scarf and subcutaneous vessels on the lower back (D)

of the right common iliac artery to $16 \mathrm{~mm}$ and the left common iliac artery to $17 \mathrm{~mm}$. There were no abnormalities in other vessels. Transthoracic echocardiography showed no abnormalities.

On physical examination, joint hypermobility (9-point Beighton scale, 6 points) and increased skin elasticity were observed. The skin was thin and shiny with signs of bruising and atrophic scars.
The patient's father (at the age of 39) and brother (at the age of 18) experienced a fatal thoracic aorta dissection. Her paternal grandmother underwent a successful operation for the ascending aortic aneurysm at the age of 66, while her 2 sisters, 1 brother, and 2 children remain asymptomatic. The relatives were not genetically tested. 
Case 2 A 26-year-old woman (height, $167 \mathrm{~cm}$; weight, $62 \mathrm{~kg}$ ) complained of hypermobility of the small joints and easy bruising. At the age of 13 , she broke her right ankle, and at the age of 19, she twisted the knee joint, and a year later she underwent a reconstruction of anterior cruciate ligament of the right knee. A recurrent ligament rupture occurred at the age of 21, and the reoperation was performed a year later. For 6 years, she had had recurrent luxation in the arm joint and the right wrist. She showed no cardiac symptoms and her transesophageal echocardiography showed only mild tricuspid insufficiency. She had never been pregnant.

On examination, joint hypermobility (9-point Beighton scale, 7 points) and a thin, translucent skin with prominent subcutaneous vessels were noted (FIGURE 1).

The patient's father died of an aortic dissection at the age of 45 . He also suffered from joint hypermobility. Her grandfather died of an aortic aneurysm rupture. The relatives were not genetically tested.

Genetic testing After obtaining written informed consent, the molecular analysis was performed on genomic DNA by polymerase chain reaction amplification of all coding exons and the flanking intron regions. The amplicons were analyzed by the sequencing-by-synthesis (SBS) technology (MiSeq Personal Sequencer, Illumina, San Diego, California, United States). The gene panel including FBN1, TGFBR1, TGFBR2, TGFB2, SMAD2, SMAD3, ACTA2, COL3A1, $M Y H 11, T G F B 3$, and SKI genes was selected based on the clinical signs and a positive family history. The presence of the variants was confirmed by Sanger sequencing. The sensitivity of SBS sequencing is more than $99.9 \%$.

We identified 2 novel COLBA1 mutations, c. $1348-2 A>G$ and c. $1455+1 G>T$ in cases 1 and 2, respectively. Both mutations disrupt the normal splice sites, thereby interfering with normal splicing. In our patient (case 1), a history of colon perforation and the iliac artery aneurysm and dissection can be considered as typical. In contrast to older patients with aneurysms, ${ }^{6}$ at a younger age, genetic syndromes associated with aortic aneurysmal disease are relatively common. ${ }^{7}$ In vEDS, spontaneous arterial rupture is most prevalent in the third and fourth decades of life and often involves thoracic or abdominal vessels as well as the descending and abdominal aorta. ${ }^{5,8}$ There is no specific treatment for vEDS; however, an early diagnosis can help monitor disease progression. Patients should be offered genetic counselling. The risk of an affected parent passing on the mutation to their child can be as high as $50 \% .^{5}$

In our second case, a recurrent ligament rupture was observed. Mutations in the COL3A1 gene have been reported to lead to this type of complications. ${ }^{9,10}$ In these cases, lifestyle changes can be recommended to minimize the risk of injuries in the future, and regular control visits and clinical surveillance are extremely important. Typical joint and skin manifestations should draw attention of a consulting cardiologist to vEDS.

Regarding reproductive history, the first patient gave birth to 2 children. However, according to the 2018 European Society of Cardiology guidelines, pregnancy in women with vEDS is not recommended (class IIIC) due to high risk of serious vascular complications. ${ }^{11}$ Murray et $\mathrm{al}^{12}$ reported that life-threatening complications occurred in $14.5 \%$ of deliveries in women with vEDS, including arterial dissection / rupture (9.2\%), uterine rupture (2.6\%), surgical complications (2.6\%), and finally, the pregnancy-related death rate of $5.3 \%$. It is unclear whether an elective cesarean section decreases mortality among such women. ${ }^{5}$

Our report clearly indicates that molecular analysis should be conducted in young patients with unexplained bowel or arterial rupture and also in patients with a family history of similar events. The 2 familial cases we presented here also illustrate the clinical variability of the disorder, which means that carriers of the same mutation may present variable clinical presentations..$^{13}$ Importantly, genetically confirmed vEDS may influence pregnancy and reproductive counseling. Pregnant women with vEDS should be considered to be at high risk of complications and they need to be referred to specialized centers. Our report also draws attention to the importance of searching for genetic causes of rare diseases in Poland, including thrombophilia. ${ }^{14,15}$ Molecular genetic analysis is an essential tool in this type of diagnostics.

\section{SUPPLEMENTARY MATERIAL}

Supplementary material is available at www.mp.pl/kardiologiapolska.

\section{ARTICLE INFORMATION}

\section{CONFLICT OF INTEREST None declared.}

OPEN ACCESS This is an Open Access article distributed under the terms of the Creative Commons Attribution-NonCommercial-NoDerivatives $4.0 \mathrm{In}$ ternational License (CC BY-NC-ND 4.0), allowing third parties to download articles and share them with others, provided the original work is properly cited, not changed in any way, distributed under the same license, and used for noncommercial purposes only. For commercial use, please contact the journal office at kardiologiapolska@ptkardio.pl.

HOW TO CITE Konieczyńska M, Wypasek E, Karpiński M, et al. Vascular Ehlers-Danlos syndrome in 2 Polish patients: identification of 2 novel COL3A1 gene mutations. Kardiol Pol. 2019; 77: 1070-1073. doi:10.33963/KP.15005

\section{REFERENCES}

1 Byers P, Belmont J, Black J, et al. Diagnosis, natural history, and management in vascular Ehlers Danlos syndrome. Am J Med Genet Part C Semin Med Genet. 2017; 175: 40-47.

2 Malfait $F$, Francomano C, Byers $P$, et al. The 2017 international classification of the Ehlers-Danlos syndromes. Am J Med Genet C Semin Med Genet. 2017; 175: 8-26.

3 Gelse K, Pöschl E, Aigner T. Collagens - structure, function, and biosynthesis. Adv Drug Deliv Rev. 2003; 55: 1531-1546.

4 Frank M, Albuisson J, Ranque B, et al. The type of variants at the COL3A1 gene associates with the phenotype and severity of vascular Ehlers-Danlos syndrome. Eur J Hum Genet. 2015; 23: 1657-1664. 
5 Pepin M, Schwarze U, Superti-Furga A, Byers PH. Clinical and genetic features of Ehlers-Danlos syndrome type IV, the vascular type. N Engl J Med. 2000; 342: 673-680.

6 Dereziński TL, Fórmankiewicz B, Migdalski A, et al. The prevalence of abdominal aortic aneurysms in the rural/urban population in central Poland -S Gniewkowo Aortic Study. Kardiol Pol. 2017; 75: 705-710.

7 Cury M, Zeidan F, Lobato AC. Disease in the young: genetic aneurysm syndromes, connective tissue disorders, and familial aortic aneurysms and dissections. JVasc Med. 2013: 267215.

8 Germain DP, Herrera-Guzman Y. Vascular Ehlers-Danlos syndrome. Ann Genet. $2004 ; 47: 1-9$.

9 Stępień-Słodkowska M, Ficek K, Maciejewska-Karłowska A, et al. Overrepresentation of the COL3A1 AA genotype in Polish skiers with anterior cruciate ligament injury. Biol Sport. 2015; 32: 143-147.

$10 O^{\prime}$ Connell K, Knight H, Ficek K, et al. Interactions between collagen gene variants and risk of anterior cruciate ligament rupture. Eur J Sport Sci. 2015; 15: 341-350.

11 Regitz-Zagrosek V, Roos-Hesselink JW, Bauersachs J, et al. 2018 ESC Guidelines for the management of cardiovascular diseases during pregnancy. Kardiol Pol. 2019; 77: 245-326.

12 Murray ML, Pepin M, Peterson S, Byers PH. Pregnancy-related deaths and complications in women with vascular Ehlers-Danlos syndrome. Genet Med. 2014; 16: 874-880.

13 Cortini F, Marinelli B, Romi S, et al. A New COL3A1 Mutation in Ehlers-Danlos syndrome vascular type with different phenotypes in the same family. Vasc Endovascular Surg. 2017; 51: 141-145.

14 Wypasek E, Corral J, Alhenc-Gelas M, et al. Genetic characterization of antithrombin, protein C, and protein $S$ deficiencies in Polish patients. Pol Arch Intern Med. 2017; 127: 512-523.

15 Bagoly Z. Uncovering the genetic background of natural anticoagulant deficiencies: time to look behind the scenes. Pol Arch Intern Med. 2017; 127: 465-467. 